\title{
Predição de ações judiciais no setor elétrico por falta de fornecimento de energia usando regressão logística
}

\author{
Dayvson W. F. Almeida* Paulo R. A. Gomes* \\ Domingos A. D. Júnior* Francisco Y. C. de Oliveira* \\ Giovanni L. F. da Silva* Darlan B. P. Quintanilha* \\ Otilio P. S. Neto* Geraldo B. Junior* André B. Cavalcante* \\ Aristófanes C. Silva* Erika W. B. A. L. Alves ${ }^{* *}$ \\ Milton S. L. de Oliveira** \\ * Núcleo de Computação Aplicada - NCA, Universidade Federal do \\ Maranhão \\ e-mail: (dayvsonalmeida, paulo.rgomes, domingos.adj, ylderlan, \\ giovannilucca, dquintanilha, otilio, geraldo, andrecavalcante, \\ ari)@nca.ufma.br \\ ** Equatorial Energia, MA \\ e-mail: (erika.assis, milton.oliveira)@equatorialenergia.com.br
}

\begin{abstract}
Understanding the complications involving the dissatisfaction of the clients of the electric power concessionaires represents the strengthening of the possibility of reversing the high degree of lawsuits against the concessionaires. Therefore, this work proposes a method based on data mining and computational intelligence to predict the issue of new lawsuits based on the lack of energy supply in the electric sector, in addition to the identification of correlated factors. The method consists of the step of data acquisition, feature engineering, feature selection, and finally, the classification step. The proposed method was validated using a private database and is robust in the task of identifying the prediction of lawsuits by means of an accuracy of $84.70 \%$; precision of $73.83 \%$; sensitivity of $60.45 \%$, specificity of $85.51 \%$ and F-score of $66.47 \%$. Thus, we demonstrate the feasibility of using the logistic regression model in conjunction with feature selection techniques to solve the problem of predicting lawsuits in the electricity sector.

Resumo: Entender as complicações que envolvem a insatisfação dos clientes das concessionárias de energia elétrica representa o fortalecimento da possibilidade de reversão do alto grau de ações judiciais. Portanto, este trabalho propõe um método para predizer ações judiciais baseado na falta de fornecimento de energia, além da identificação de fatores correlacionados. O método é composto pela etapa de aquisição dos dados, engenharia de característica, seleção de características, e por fim, a etapa de classificação. O método proposto foi validado utilizando um banco de dados de uma empresa privada e mostra-se robusto na tarefa de predizer ações judiciais por meio de uma acurácia de $84,70 \%$, precisão de $73,83 \%$, sensibilidade de $60,45 \%$, especificidade de $85,51 \%$ e $F$-Measure de $66,47 \%$. Assim, demonstramos a viabilidade de usar o modelo de regressão logística em conjunto com técnicas de seleção de características para resolver o problema da predição de processos judiciais.
\end{abstract}

Keywords: Lawsuit; electricity sector; customer profile; data mining; computational intelligence; logistic regression.

Palavras-chaves: Ação judicial; setor elétrico; perfil do cliente; mineração de dados; inteligência computacional; regressão logística.

\section{INTRODUÇÃO}

Os serviços prestados por concessionárias de energia elétrica têm sido cada vez mais objeto de insatisfação por parte dos consumidores brasileiros. O desconhecimento detalhado de características vinculadas às causas desta insatisfação compromete o processo de planejamento de ações que venham modificar este cenário (Borges and Lima, 2013). Por se tratar de um setor cujo negócio principal é baseado em prestação de serviços, impõe-se uma dificul- dade específica já que é improvável a eliminação total das falhas na prestação de serviço e porque os consumidores têm expectativas diferentes com relação a diferentes níveis de falhas (Johnston and Fern, 1999).

Segundo Almeida et al. (2014), as despesas com indenizações de clientes, custas processuais, honorários periciais, gastos com escritórios e com estrutura jurídica representaram $60 \%$ do valor de perda decorrentes da insatisfação do cliente e os $40 \%$ restantes com despesas decorrentes 
do mau serviço, tais como vistorias e reexecuções, gastos com clientes, por exemplo o custo de ressarcimento de aparelhos, e multas por descumprimento de regulação.

O entendimento das complicações que envolvem a insatisfação dos consumidores representa o fortalecimento de possibilidades de reversão do alto grau de insatisfação verificado junto a estas concessionárias. Portanto, esse trabalho propõe investigar a propensão do cliente à proposição de ações judiciais em relação ao serviço de fornecimento de energia. Uma vez que a falta de fornecimento de energia é uma das maiores causas de processos contra a concessionária grupo Equatorial Energia, responsável pela distribuição de energia elétrica nos estados do Pará, Maranhão, Piauí e Alagoas, possuindo mais de 5 milhões de clientes no Brasil.

O método proposto nesse trabalho utiliza técnicas de mineração de dados e inteligência computacional para predizer quais clientes possuem propensão à entrar com uma ação judicial contra a Equatorial Energia pela causa de falta de fornecimento de energia. O método é composto por quatro etapas. A primeira etapa consiste na aquisição dos dados dos clientes. Na segunda etapa, a engenharia de característica é executada, nessa etapa explicamos as características extraídas do banco de dados e como as novas características são construídas. Depois, na terceira etapa, a seleção de características é executada. Por fim, a classificação é realizada usando o modelo de regressão logística.

\section{TRABALHOS RELACIONADOS}

A identificação de clientes que tenham propensão de entrar com uma ação judicial contra a empresa é considerada como uma identificação de insatisfação do cliente. A empresa sofre uma perda considerável de receita quando alguns clientes a deixam, além dos custos processuais gerados. Tal finalidade é classificada como Customer Churn Prediction (CCP), onde técnicas baseadas em aprendizado de máquina, análises de regressão e modelagem preditiva são usadas para estimar a probabilidade dos clientes deixarem a empresa. O CCP é um problema comum, não apenas em empresas de distribuição de energia, mas também de outros setores como telecomunicações (Keramati et al., 2014; Amin et al., 2019), bancário (He et al., 2014), e comércio eletrônico (Gordini and Veglio, 2017).

He et al. (2014) prevêem a rotatividade de clientes de banco comercial com base no modelo de máquinas de vetores de suporte (SVM) e usa o método de amostragem aleatória para melhorar o modelo SVM. Gordini and Veglio (2017) desenvolveram um modelo sob medida para a indústria de comércio eletrônico B2B baseada em SVM. Seus resultados experimentais mostraram que o SVM supera a regressão logística e as redes neurais.

Keramati et al. (2014) preveem a rotatividade de clientes em uma empresa de telecomunicações utilizando árvores de decisão, redes neurais artificiais e k vizinhos mais próximos, onde os resultados mostraram a superioridade das redes neurais artificiais. Amin et al. (2017) propõe uma técnica para tomada de decisão baseada na teoria dos conjuntos aproximados (TCA). Os autores avaliaram o desempenho da CCP baseada em TCA usando quatro algoritmos de geração de regras: exaustivo, genético, cove- ring e o LEM2 (Learning from Examples Module, versão 2). A classificação de TCA baseada em algoritmo genético supera outros algoritmos de geração de regras utilizados. Posteriormente, os autores propuseram uma nova abordagem de CCP baseada no conceito de estimativa de certeza do classificador usando o fator de distância (Amin et al., 2019).

Os trabalhos citados não se tratam de identificação de clientes com propensão de entrar com uma ação judicial, e sim sobre a satisfação de clientes e de suas rotatividades na empresa. Todavia, foram fundamentais para o embasamento deste trabalho, mesmo não sendo possível fazer comparação com o método proposto. Verifica-se que não há um método específico que possua sempre o melhor desempenho nas diferentes propostas, mesmo que todas sejam consideradas propostas de CCP.

\section{MÉTODO PROPOSTO}

O método proposto para predição de processos judiciais por falta de energia no setor elétrico é dividido em quatro etapas. Em resumo, o primeiro passo consiste no processo de aquisição de dados. Na segunda etapa, a engenharia de característica é executada, nessa etapa explicamos as características extraídas do banco de dados e como as novas características são construídas. Então, na terceira etapa, a seleção de características é executada. Finalmente, a previsão é realizada usando o modelo de regressão logística.

\subsection{Aquisição dos dados}

O banco de dados usado neste trabalho é uma base privada obtida da Companhia Energética do Maranhão (CEMAR). Esses dados incluem informações como histórico de consumo, alteração de consumo, mudança repentina no consumo, duração de falta de energia, comportamento judicial do cliente, informações financeiras, notas de serviços e reclamações feitas pelo cliente, entre outras informações. Assim, percebe-se que as informações de perfil de um cliente estão espalhadas por vários domínios.

No banco de dados da concessionária, os dados desejados são distribuídos em várias tabelas. A ferramenta Talend Open Studio Data Integration foi utilizada para unir as várias tabelas e gerar arquivos com valores separados por vírgulas (.csv). Após a aquisição dos dados, o próximo passo é avaliar quais características das tabelas serão usadas para a construção do modelo preditivo.

\subsection{Engenharia de característica}

A engenharia de característica é o processo de usar o conhecimento do domínio dos dados para criar características que permitem com que os algoritmos de aprendizado de máquina funcionem (Turner et al., 1999). O seguinte conjunto de características foi extraído do banco de dados:

(1) Informações gerais: características individuais de cada cliente, tais como localização (latitude e longitude) e tipo de cliente (residencial, comercial ou rural).

(2) Consumo de energia: características que lidam com o perfil de consumo de cada cliente, tais como consumo registrado e leitura faturada. 
(3) Perda de energia: características relacionadas à perda de energia de um transformador conectado a um cliente. As informações adquiridas foram de frequência de perda de energia, duração da perda, informação se o cliente possui equipamentos especiais que não poderia ser desligados

(4) Histórico de desligamentos: características relacionadas ao desligamento do fornecimento de energia dos clientes.

(5) Faturas: características que mostram o histórico de pagamento de faturas.

(6) Jurídica: características relacionadas a ações judiciais anteriores tomadas pelo cliente. Essa informação é usada durante o treinamento para informar as classes.

É importante notar que as características extraídas do banco de dados vêm em uma forma grosseira, às vezes categórica e não normalizada. Assim, é importante aplicar uma transformação nos dados. Para isso, nós utilizamos a ferramenta Featuretools (Kanter and Veeramachaneni, 2015). As transformações de características usadas neste trabalho são descritas a seguir: normalização das variáveis numéricas, codificação one-hot, geração de estatísticas como média e desvio padrão, e por fim, extração dos dados baseado em diferentes intervalos de tempos.

\subsection{Seleção de característica}

A seleção de característica é o problema de selecionar um subconjunto de $N$ características a partir de um conjunto de $D$ características com base em técnicas de otimização. O principal objetivo da seleção de característica é projetar um classificador mais compacto com pouca ou nenhuma degradação de desempenho (Xue et al., 2016). Nesse trabalho, duas técnicas de seleção de característica são avaliadas separadamente, o algoritmo de otimização de enxame de partículas (PSO) (Eberhart and Kennedy, 1995) e o algoritmo recursivo de eliminação de características (RFE) (Guyon et al., 2002).

O algoritmo de otimização de enxame de partículas (PSO) é uma técnica evolutiva inspirada pelo comportamento de enxame ou colaborativo de populações biológicas (Eberhart and Kennedy, 1995). O PSO procura uma solução ideal alterando iterativamente a velocidade e a posição com base na experiência de voo da própria partícula e do grupo em direção à localização de Gbest e Pbest em iterações sucessivas. O Gbest corresponde ao melhor valor de aptidão da população que qualquer partícula alcançou enquanto o Pbest corresponde ao melhor valor de aptidão da partícula que alcançou até o momento (Kennedy, 2010).

$$
\text { Fitness }=\alpha(1-\text { Sensibilidade })+(1-\alpha)\left(1-\frac{N}{D}\right)
$$

onde $\alpha$ um hiper-parâmetro que decide a troca entre a métrica sensibilidade e o tamanho do subconjunto de características $N$ em relação ao número total de características $D$ no conjunto de dados Vieira et al. (2013).

O algoritmo recursivo de eliminação de características (RFE) foi proposto por Guyon et al. (2002) para a seleção de genes em um problema de classificação usando máquinas de vetores de suporte (SVM). Um bom critério de ranqueamento de contribuição de características não é necessariamente um bom critério de seleção de subconjunto de destas características. Assim, dado um estimador externo que pesa as características de um problema (por exemplo, SVM, regressão linear, entre outros), o RFE tem objetivo de selecionar as características recursivamente a fim de se obter um subconjunto de características cada vez menor.

O estimador externo é treinado com um conjunto inicial de características e a importância de cada característica é ponderada pela atribuição de um coeficiente que o estimador define para cada uma. As características menos importantes são removidas do conjunto atual a cada iteração, até que o número desejado de características selecionadas seja atingido (Guyon et al., 2002).

\subsection{Classificação}

Para a construção do modelo preditivo foi utilizado a técnica de Regressão Logística. A regressão logística é uma técnica estatística que tem como objetivo produzir, a partir de um conjunto de variáveis, um modelo que permita a predição de valores tomados por uma variável categórica, frequentemente binária, a partir de uma série de variáveis explicativas (Brito and Neto, 2008). Na regressão logística, há uma transformação na variável dependente, que é convertida em uma razão de probabilidades e posteriormente em uma variável de base logarítmica (transformação logística). Devido à natureza não linear dessa transformação, os coeficientes da regressão são estimados pelo método da máxima verossimilhança. O modelo da regressão logística assume a seguinte relação:

$$
\ln \left(\frac{p}{1-p}\right)=b_{0}+b_{1} X_{1}+b_{2} X_{2}+\ldots+b_{k} X_{k}
$$

em que $p$ é a probabilidade de ocorrer o evento, $1-p$ a probabilidade de não ocorrer o evento, $p /(1-p)$ a razão de probabilidades, $X_{i}$ as variáveis independentes e $b_{i}$ os coeficientes estimados.

Nesse trabalho, nós utilizamos o modelo de regressão logística tanto na etapa de seleção de características juntamente com o algoritmo do PSO e o algoritmo de RFE, quanto na etapa de classificação. A escolha por essa técnica deve-se a facilidade para lidar com variáveis independentes categóricas, fornece resultados em termos de probabilidade, alto grau de confiabilidade e requer pequeno número de suposições (Brito and Neto, 2008).

\subsection{Métricas de avaliação}

Finalmente, é necessário validar a metodologia. Este trabalho utiliza estatísticas comumente usadas em problemas de classificação binária para verificar o desempenho da metodologia em identificar os consumidores que possuem propensão de entrar com uma ação judicial contra a empresa por falta de fornecimento de energia. As métricas utilizadas foram acurácia, precisão, sensibilidade, especificidade e a F-Measure (Amin et al., 2017).

\section{RESULTADOS E DISCUSSÃO}

Esta seção apresenta e discute os resultados obtidos com o método proposto para predição de ações judiciais por falta 
de fornecimento de energia. Para avaliação, o banco de dados adquirido foi dividido em dois conjuntos: treinamento e teste. Os consumidores que tiveram falta de energia foram obtidos a partir de dados históricos de ações judiciais com esse assunto. Consumidores sem falta de energia foram selecionados aleatoriamente entre outros consumidores. Foi mantida uma proporção de 1 para 3 entre consumidores que entraram e não entraram com ações judiciais por falta de fornecimento de energia, a fim de realizar experimentos com dados desequilibrados que são esperados para o problema. Um total de 13.576 consumidores foram analisados. A proporção entre consumidores com e sem ações judiciais no conjunto de dados de treinamento e teste é descrita na Tabela 1.

Tabela 1. Proporção entre clientes com e sem processos de ação judicial (PAJ) contra empresa por falta de fornecimento de energia.

\begin{tabular}{cccc}
\hline Base & Cliente com PAJ & Cliente sem PAJ & Total \\
\hline Treino & 2.035 & 6.099 & 8.134 \\
Teste & 1.360 & 4.082 & 5.442 \\
Total & 3.395 & 10.181 & 13.576 \\
\hline
\end{tabular}

Podemos observar que a base de dados é heterogênea quando se trata do número de consumidores. Mais de treze mil consumidores são avaliados na metodologia proposta. Há um desequilíbrio entre as classes tanto na criação do modelo em treinamento quanto no teste.

Após a etapa de engenharia de características (Subseção 3.2), 1171 características relacionadas a falta de fornecimento de energia foram extraídas. A próxima etapa consiste na seleção de características (Subseção 3.3). Nesta etapa, os algoritmos PSO e RFE são usados para selecionar as melhores características entre as 1171 extraídas da base de dados.

Finalmente, na etapa de classificação (Subseção 3.4) usase o conjunto de dados de treinamento para prever consumidores do conjunto de dados de teste que entraram com ação judicial por problemas relacionados a falta de fornecimento de energia. A Tabela 2 resume os resultados obtidos pela metodologia, incluindo acurácia (Acc), precisão (Prec), sensibilidade (Sen), especificidade (Esp) e a $F$-Measure no conjunto de teste.

De acordo com a Tabela 2, os melhores resultados foram obtidos usando o modelo de regressão logística em conjunto com o algoritmo RFE apresentando uma acurácia de $84,70 \%$, precisão de $73,83 \%$, sensibilidade de $60,45 \%$, especificidade de $85,51 \%$ e F-Measure de $66,47 \%$. Além disso, o número de características reduziu bastante em comparação com o experimento com a quantidade de características original, 1171 para 600 características. Em relação à métrica de precisão, o modelo de regressão logística com seleção de características baseada no algoritmo PSO alcançou o maior desempenho, apresentando uma precisão de $74,93 \%$.

Para verificar a eficiência do regressor logístico na tarefa de predição dos clientes que possuem propensão a entrar com processos judiciais por falta de energia no setor elétrico, nós utilizamos outras técnicas de aprendizado de máquina comumente aplicadas na tarefa de mineração de dados. As técnicas utilizadas foram o XGBoost (Chen and Guestrin, 2016), o Random Forest (Breiman, 2001) e a rede profunda
LSTM (Hochreiter and Schmidhuber, 1997). A Tabela 3 apresenta os resultados obtidos por todas as técnicas no mesmo conjunto de teste. A única técnica que obteve uma acurácia maior que o regressor logístico foi o modelo do XGBoost. No entanto, sua sensibilidade foi abaixo e o tempo de treinamento foi bem mais demorado.

Importante destacar que neste tipo de aplicação deseja-se obter o maior número possível de indivíduos com predisposição para uma ação judicial, métrica destacada pela sensibilidade da abordagem. Esses indivíduos ajudam a identificar fatores comuns que estão influenciando e podem ser usados pela companhia como fator de melhoria dos serviços prestados. Também destaca-se que muitos clientes que foram anotados como sem processo podem estar em situações semelhantes aqueles que entraram com a ação, mas que todavia, não se mostraram ainda interessados em realizá-la.

Após a análise quantitativa dos resultados, o framework LIME (Ribeiro et al., 2016) foi aplicado para a análise das características relevantes na predição do modelo treinado. Para isso, nós utilizamos o modelo do regressor logístico com a seleção de características baseado no algoritmo RFE, devido ao melhor resultado e o menor número de características. A Figura 1 apresenta a explicação da predição de um cliente com processo classificado corretamente (VP) com as cinco características mais relevantes. As probabilidades da predição foram de $100 \%$ para a classe processo e $0 \%$ para a classe sem processo. Dentre as cinco mais relevantes para a predição desse cliente, percebe-se que quatro das cinco características foram geradas na etapa de engenharia (Subseção 3.2), evidenciando a importância dessa etapa no fluxograma da metodologia proposta.

A Figura 2 mostra a explicação da predição de um cliente com processo classificado erroneamente (FN) com suas cinco características mais relevantes. As probabilidades da predição foram de $22 \%$ para a classe processo e $78 \%$ para a classe sem processo. Nota-se também que apenas a característica Latitude foi extraída do banco de dados na empresa, as demais foram geradas na etapa de engenharia. Comparando-o com a Figura 1 apenas as características COUNT(desligamentos) e COUNT(desligamentos WHERE STATUS DOC SUSP = ENCERRADO DEFINITIVAMENTE) se repetem na explicação. Contudo, não foram suficientes para o acerto do cliente como cliente com processo.

A Figura 3 apresenta a explicação da predição de um cliente sem processo classificado corretamente (VN) com as cinco características mais relevantes. As probabilidades da predição foram de $96 \%$ para a classe processo e $4 \%$ para a classe sem processo. E por fim, a Figura 4 ilustra a explicação da predição de um cliente sem processo classificado erroneamente (FP) com as cinco características mais relevantes. As probabilidades da predição foram de $23 \%$ para a classe processo e $77 \%$ para a classe sem processo.

Baseado nas explicações das predições dos clientes, percebese a importância da etapa de engenharia de características na metodologia, uma vez que as características geradas ocupam a maior parte das características mais relevantes. 
Tabela 2. Resultados da predição de ações judiciais usando regressão logística com e sem seleção de características.

\begin{tabular}{|c|c|c|c|c|c|c|}
\hline Experimentos & Características & Acc $(\%)$ & Prec (\%) & Sen $(\%)$ & Esp (\%) & F-Measure (\%) \\
\hline Sem seleção de características & 1171 & 84,66 & 73,78 & 60,30 & 92,82 & 66,37 \\
\hline Seleção de características (PSO) & 657 & 82,38 & 74,93 & 44,75 & 83,69 & 56,04 \\
\hline Seleção de características (RFE) & 600 & 84,70 & 73,83 & 60,45 & 85,51 & 66,47 \\
\hline
\end{tabular}

Tabela 3. Resultados da predição de ações judiciais usando regressão logística e outras técnicas de aprendizado de máquina.

\begin{tabular}{lccccc}
\hline \multicolumn{1}{c}{ Técnica } & Acc (\%) & Prec (\%) & Sen (\%) & Esp (\%) & F-Measure (\%) \\
\hline XGBoost & 87,37 & 85,55 & 59,81 & 96,61 & 70,40 \\
Random Forest & 84,33 & 81,44 & 48,64 & 96,28 & 60,90 \\
LSTM & 84,22 & 81,78 & 47,44 & 96,43 & 60,30 \\
Regressor logístico com RFE & $\mathbf{8 4 , 7 0}$ & $\mathbf{7 3 , 8 3}$ & $\mathbf{6 0 , 4 5}$ & $\mathbf{8 5 , 5 1}$ & $\mathbf{6 6 , 4 7}$ \\
\hline
\end{tabular}

Probabilidades
Sem Processo 0.00
Processo $\square$

Sem Processo

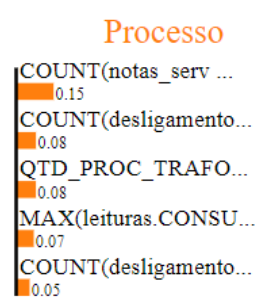

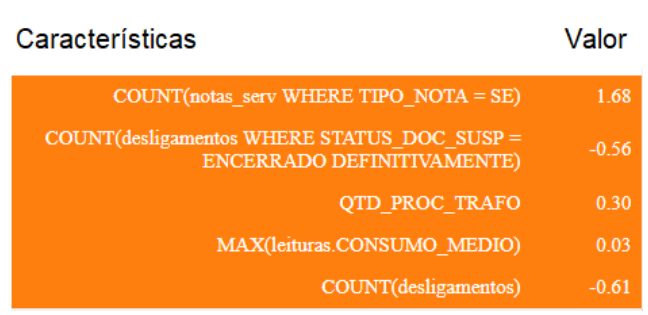

Figura 1. Explicação da predição de um cliente com processo classificado corretamente.
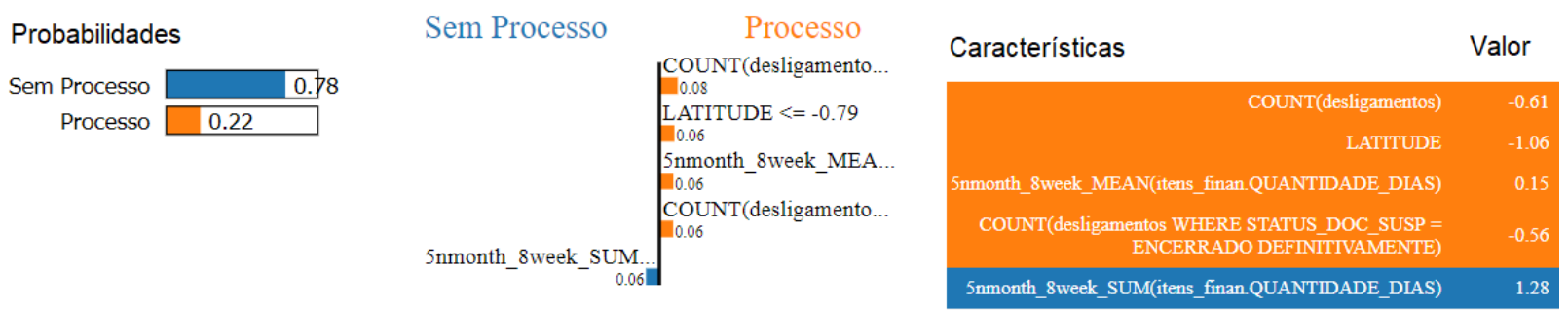

Figura 2. Explicação da predição de um cliente com processo classificado erroneamente.
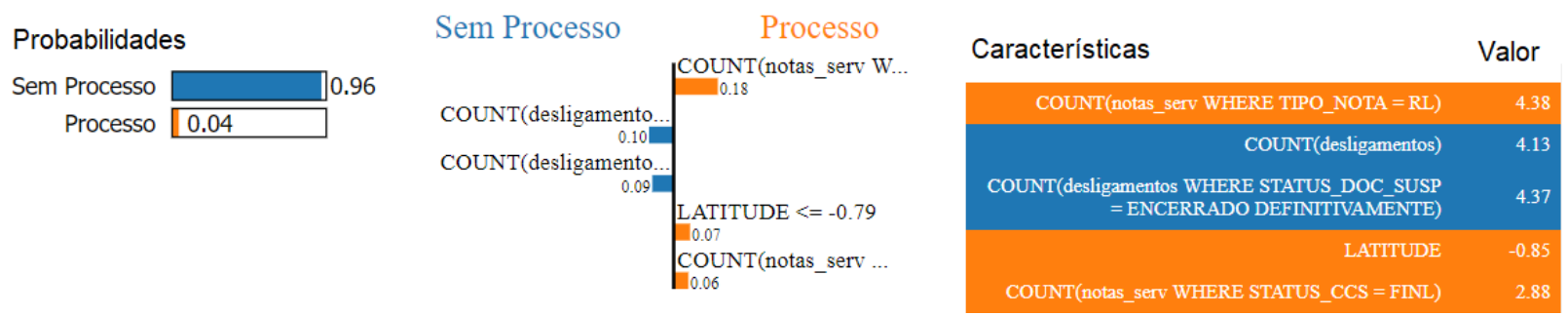

Figura 3. Explicação da predição de um cliente sem processo classificado corretamente.

\section{CONCLUSÃO}

Este trabalho investigou o desempenho do modelo de regressão logística em conjunto com técnicas de seleção de características (PSO e RFE) para predição de processos judiciais por falta de energia no setor elétrico com base nos dados do cliente. Os melhores resultados foram obtidos utilizando o modelo de regressão logística em conjunto com o algoritmo RFE apresentando uma acurácia de 84,70\%, precisão de $73,83 \%$, sensibilidade de $60,45 \%$, especificidade de $85,51 \%$ e $F$-Measure de $66,47 \%$.

No futuro, pretendemos investigar novas abordagens que possam melhorar a eficácia do método proposto. Em primeiro lugar, a base de dados é desbalanceada, clientes que entraram na com ação judicial por conta da falta de fornecimento de energia (classe minoritária) contém um número menor de amostras em comparação com os clientes sem ação judicial (classe majoritária). Em segundo lugar, a eliminação e a detecção de outliers auxiliariam muito 

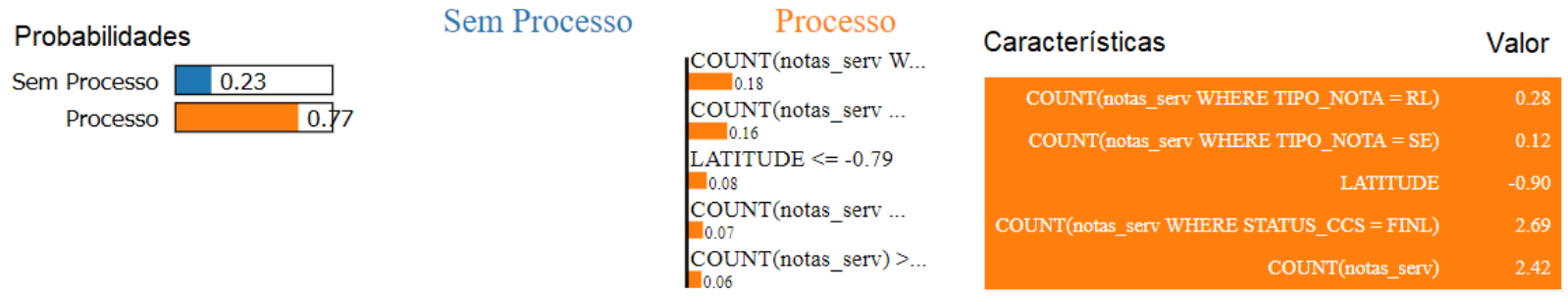

Figura 4. Explicação da predição de um cliente sem processo classificado erroneamente.

para fornecer melhores resultados. Finalmente, analisar outras técnicas de seleção de característica e fazer uma comparação mais detalhada.

\section{AGRADECIMENTOS}

Este trabalho foi apoiado pelo projeto Sipaju, financiado pela Equatorial Energia e pela Agência Nacional de Energia Elétrica (ANEEL) através do P\&D No PD-00037$0031 / 2018$.

\section{REFERÊNCIAS}

Almeida, V.M.C.d., Figueiredo, O.H.d.S., and Silva, G.F.d. (2014). Propensão do cliente à proposição de ações judiciais: Proposição e teste de modelo preditivo para o setor de energia elétrica. Revista Brasileira de Marketing, 13(5), 01-18.

Amin, A., Al-Obeidat, F., Shah, B., Adnan, A., Loo, J., and Anwar, S. (2019). Customer churn prediction in telecommunication industry using data certainty. Journal of Business Research, 94, 290 - 301. doi:https://doi.org/10.1016/j.jbusres.2018.03. 003. URL http://www. sciencedirect.com/science/ article/pii/S0148296318301231.

Amin, A., Anwar, S., Adnan, A., Nawaz, M., Alawfi, K., Hussain, A., and Huang, K. (2017). Customer churn prediction in the telecommunication sector using a rough set approach. Neurocomputing, 237, 242 - 254. doi:https://doi.org/10.1016/j.neucom.2016.12. 009. URL http://www.sciencedirect.com/science/ article/pii/S0925231216314849.

Borges, F.Q. and Lima, M.A.S. (2013). Insatisfação do consumidor: Uma análise nos serviços prestados por concessionárias de energia elétrica. Perspectivas Contemporâneas, 8(1).

Breiman, L. (2001). Random forests. Machine learning, 45(1), 5-32.

Brito, G.A.S. and Neto, A.A. (2008). Modelo de classificação de risco de crédito de empresas. Revista Contabilidade \& Finanças, 19(46), 18-29.

Chen, T. and Guestrin, C. (2016). Xgboost: A scalable tree boosting system. In Proceedings of the 22nd acm sigkdd international conference on knowledge discovery and data mining, 785-794. ACM.

Eberhart, R. and Kennedy, J. (1995). Particle swarm optimization. In Proceedings of the IEEE international conference on neural networks, volume 4, 1942-1948. Citeseer.

Gordini, N. and Veglio, V. (2017). Customers churn prediction and marketing retention strategies. an application of support vector machines based on the auc parameter- selection technique in b2b e-commerce industry. Industrial Marketing Management, 62, 100-107.

Guyon, I., Weston, J., Barnhill, S., and Vapnik, V. (2002). Gene selection for cancer classification using support vector machines. Machine learning, 46(1-3), 389-422.

He, B., Shi, Y., Wan, Q., and Zhao, X. (2014). Prediction of customer attrition of commercial banks based on svm model. Procedia Computer Science, 31, 423 - 430. doi:https://doi.org/10.1016/j.procs.2014.05. 286. URL http://www.sciencedirect.com/science/ article/pii/S1877050914004633. 2nd International Conference on Information Technology and Quantitative Management, ITQM 2014.

Hochreiter, S. and Schmidhuber, J. (1997). Long shortterm memory. Neural computation, 9(8), 1735-1780.

Johnston, R. and Fern, A. (1999). Service recovery strategies for single and double deviation scenarios. Service Industries Journal, 19(2), 69-82.

Kanter, J.M. and Veeramachaneni, K. (2015). Deep feature synthesis: Towards automating data science endeavors. In 2015 IEEE International Conference on Data Science and Advanced Analytics, DSAA 2015, Paris, France, October 19-21, 2015, 1-10. IEEE.

Kennedy, J. (2010). Particle swarm optimization. Encyclopedia of machine learning, 760-766.

Keramati, A., Jafari-Marandi, R., Aliannejadi, M., Ahmadian, I., Mozaffari, M., and Abbasi, U. (2014). Improved churn prediction in telecommunication industry using data mining techniques. Applied Soft Computing, 24, 994-1012.

Ribeiro, M.T., Singh, S., and Guestrin, C. (2016). "why should I trust you?": Explaining the predictions of any classifier. In Proceedings of the 22nd ACM SIGKDD International Conference on Knowledge Discovery and Data Mining, San Francisco, CA, USA, August 13-17, 2016, 1135-1144.

Turner, C.R., Fuggetta, A., Lavazza, L., and Wolf, A.L. (1999). A conceptual basis for feature engineering. Journal of Systems and Software, 49(1), 3-15.

Vieira, S.M., Mendonça, L.F., Farinha, G.J., and Sousa, J.M. (2013). Modified binary pso for feature selection using svm applied to mortality prediction of septic patients. Applied Soft Computing, 13(8), 3494-3504.

Xue, B., Zhang, M., Browne, W.N., and Yao, X. (2016). A survey on evolutionary computation approaches to feature selection. IEEE Transactions on Evolutionary Computation, 20(4), 606-626. 